July 1995

PAR-LPTHE 95-38

ULB-TH 10/95

hep-th/9507061

\title{
BLACK HOLE ENTROPY AND STRING INSTANTONS
}

\author{
François Englert Đ \\ Service de Physique Théorique \\ Université Libre de Bruxelles, Campus Plaine, C.P. 225 \\ Boulevard du Triomphe, B-1050 Bruxelles, Belgium \\ Laurent Houart [2 and Paul Windey \\ Laboratoire de Physique Théorique et Hautes Energies t \\ Université Pierre et Marie Curie, Paris VI \\ Bte 126, 4 Place Jussieu, 75252 Paris cedex 05, France
}

\begin{abstract}
The coupling of a string to gravity allows for Schwarzschild black holes whose entropy to area relation is $S=(A / 4)(1-4 \mu)$, where $\mu$ is the string tension. This departure from the A/4 universality results from a string instanton generating a black hole with smaller entropy at a temperature exceeding the Hawking value. The temperature is sensitive to the presence of classical matter outside the black hole horizon but the entropy is not. The horizon materializes at the quantum level. It is conjectured that such a macroscopic non local effect may be operative in retrieving information from incipient black holes.
\end{abstract}

\footnotetext{
${ }^{1}$ e-mail: fenglert@ulb.ac.be

${ }^{2}$ Chercheur associé CNRS, e-mail: lhouart@lpthe.jussieu.fr

${ }^{3}$ e-mail: windey@lpthe.jussieu.fr

${ }^{4}$ Laboratoire associé No. 280 au CNRS.
} 


\section{Introduction}

In absence of back reaction, an incipient black hole of mass $M$ emits a thermal flux at the Hawking temperature $\beta_{H}^{-1}$ given by [1]

$$
\beta_{H}=8 \pi M
$$

The flux is filtered by the centrifugal barrier and consists predominantly of low angular momentum quanta which have leaked out and escape towards infinity.

To study the thermodynamic properties of the black hole, one may consider, instead of its irreversible evaporation, reversible exchanges between the black hole and surrounding matter in a state of thermal equilibrium. Assuming that $M$ is equal to its thermodynamic energy $E$, one may characterize such exchanges by

$$
d S=\beta_{H} d M
$$

where $S$ is the black hole entropy. This gives, up to a constant of integration, the Bekenstein-Hawking [2] area entropy namely

$$
S=\frac{A}{4}
$$

where $A=16 \pi M^{2}$ is the area of the event horizon.

The entropy Eq. (3) is independent of the particular collapse and is therefore expected to be a genuine property of the Schwarzschild background geometry. To display this property, it is convenient to extend maximally the original Schwarzschild metric. The extended metric describes an "eternal" black hole which is time reversal symmetric and is therefore suitable for describing thermal equilibrium. This extension introduces, in addition to the dynamical regions separated from the Schwarzschild static patch by future and past horizons, a copy of the original Schwarzschild patch. When referring to a Schwarzschild background, we shall always mean the original patch.

To understand how entropy can hide in the geometry, we first recall that to compute thermal correlation functions and partition functions in field theory in flat Minkowski space-time one can use path integrals in periodic imaginary time. The period $\beta$ is the inverse temperature and can be chosen freely. This method was generalized to compute matter correlation functions in static curved backgrounds. For the Schwarzschild background, the 
analytic continuation to imaginary time defines an Euclidean manifold everywhere except at the analytic continuation of the horizon, namely the 2-sphere at $r=2 M, M$ being the black hole mass. This ambiguity is usually removed by assuming that the Euclidean manifold is regular at $r=2 M$. The Euclidean black hole obtained in this way has a periodicity $\beta$ uniquely defined in terms of the black hole mass. The relation is $\beta=8 \pi M$ and gives back the temperature of the Hawking radiation Eq. (1).

Gibbons and Hawking [3] extended the analytic continuation to the gravitational action, restricting the hitherto ill-defined path integral over metrics to a saddle point in the Euclidean section. To constitute such a saddle the Euclidean black hole must be regular given that a singularity at $r=2 M$ would invalidate the solution of the Euclidean Einstein equations. The partition function evaluated on this saddle is interpreted as $e^{-\beta F}$, where $\beta$ is equal to $\beta_{H}$ and $F$ the free energy of the background geometry. This yields back the Bekenstein-Hawking area entropy Eq. (3).

The Gibbons-Hawking procedure gives the expected thermodynamic results but its justification is not obvious. Were it not for the saddle condition, the functional integral over metrics would formally amount to a computation of a partition function. However, not only is the Euclidean functional integral over all metrics meaningless but the partition function of the canonical ensemble for a black hole whose density of states would be given by the exponential of the entropy Eq. (3) does not exist because of the divergence at large masses. Clearly, this divergence is cut off by the saddle and we shall give a thermodynamic argument to justify this procedure.

The saddle prescription can be used to show that the relation Eq. (1) between the temperature and the black hole mass is affected by classical surrounding matter but that the entropy remains unchanged and is still given by Eq. (3). This value of the entropy seems therefore to depend only on the black hole mass. This is not the case. A different relation between entropy and area emerges when a conical singularity is present in the Euclidean section at $r=2 M$ 田.

Many authors 15, 6, 7, 8, 9, 10 have introduced a conical singularity at $r=2 M$. This modifies the Euclidean periodicity of the black hole and therefore the temperature. However if the source producing this singularity is not taken into account, the Euclidean black hole solution is not a saddle point of the functional integral and such a procedure cannot be used to find the thermodynamic properties of the background. A true Euclidean saddle 
point can nonetheless be maintained by introducing a string term in the action. The string is treated in first quantization. One may interpret this as a fundamental string term or alternatively as a thin vortex limit of a Nielsen-Olesen flux tube in the spontaneously broken phase of a field theory. The conical singularity arises from a string "instanton" wrapping around the Euclidean continuation of the horizon and the associated deficit angle is determined by the string tension. The temperature varies continuously above the Hawking value as a function of the string tension but, as shown in reference [4], it now necessarily entails a variation of the entropy versus area ratio: this ratio takes value in the interval [0,1/4], the lower limit being approached when the cone degenerates.

In this paper we generalize this result and discuss its significance. In Section 2 we review for completeness the results of reference [4. We introduce by hand a Nambu-Goto string in the action and require, as a working hypothesis - to be justified in the Section 4 - that the Schwarzschild black hole solutions analytically continued to imaginary time satisfy the Euclidean Einstein equations. This requirement fixes uniquely the Euclidean periodicity in terms of the string tension for a black hole of mass $M$ in the presence of a Euclidean string "instanton" wrapped once around the horizon. We then give the modification of the relation between entropy and area resulting from the change in periodicity induced by the string. This comes about because the product of the temperature and the entropy, a purely classical quantity, retains its traditional Schwarzschild value independently of the string tension. In Section 3 we show that, at fixed string tension, the relation between entropy and area in presence of the string instanton is, in contradistinction with the temperature, insensitive to the introduction of classical surrounding matter. In Section 1 a thermodynamic argument is presented to justify the working hypothesis used throughout the article. It leads to the necessary introduction of the string if the Euclidean periodicity is chosen to be different from its conventional value. It also fixes the value of the string tension to be given by the Euclidean saddle constraint. We then confront the problem posed by the existence of several Euclidean saddles, namely those arising from configurations with and without a string wrapping once, and possibly many times, around the 2-sphere $r=2 M$ in the Euclidean section. We conclude that while the no instanton solution defines a stable black hole with minimal temperature and maximal entropy $A / 4$, the other solutions correspond to thermodynamically distinct overheated black holes with lower 
entropy. In Section 5 we show, in a simplified model, that the string instanton induces on the horizon, in the Lorentzian background, singularities in the energy-momentum tensor of quantum fields at the loop level. This amounts to a materialization of the horizon at the quantum level. We argue that a similar situation may arise for incipient black holes and we suggest that such a non local quantum effect may play a crucial role in retrieving the information hidden by, or stored on the horizon.

\section{The String Instanton}

The Lorentzian action for gravity coupled to matter fields is taken to be

$$
I=\frac{1}{16 \pi} \int_{M} \sqrt{|g|} R-\frac{1}{8 \pi} \int_{\partial M} \sqrt{|h|} K+I_{\text {matter }}
$$

Here $\frac{1}{16 \pi} \int_{M} \sqrt{|g|} R$ is the usual Einstein-Hilbert action, $K$ is the trace of the extrinsic curvature on the boundary $\partial M$ of the four dimensional manifold $M$, and $h$ the determinant of the induced metric.

The introduction of the $K$-term requires explanation. We will justify it briefly and refer the interested reader to the recent detailed discussion of Hawking and Horowitz [1]. The Einstein-Hilbert action contains secondorder derivatives of the metric. If the system evolves between two non intersecting spacelike hypersurfaces these second derivative terms can be transformed by partial integration into boundary terms on these spacelike surfaces and on timelike surfaces. Explicitly these boundary terms stem from the integral of the four-divergence $\partial_{\mu} \omega^{\mu}$ where

$$
\omega^{\mu}=-\frac{1}{16 \pi}\left(\partial_{\nu}\left(\sqrt{|g|} g^{\mu \nu}\right)+g^{\mu \nu} \partial_{\nu} \sqrt{|g|}\right) .
$$

Their contribution to the action Eq. (1) is cancelled by the $K$-term. The absence of boundary terms on the spacelike surfaces is necessary for the consistency of the Hamiltonian formalism. However, for the asymptotically flat spaces considered here, the $K$-term introduces divergences at spacelike infinity. These can be removed by subtracting from Eq. (丑) a $K$-term at infinity in flat space. It can then be verified that the subtracted action

$$
I-I_{0}=\frac{1}{16 \pi} \int_{M} \sqrt{|g|} R-\frac{1}{8 \pi} \int_{\partial M} \sqrt{|h|} K
$$




$$
+\frac{1}{8 \pi} \int_{(\partial M)_{\infty}} \sqrt{\left|h_{0}\right|} K_{0}+I_{\text {matter }}
$$

yields the correct ADM mass as the on-shell value of the Hamiltonian. The action Eq. (6) can now be written as a Hamiltonian action and the path integral over metrics can be formally defined.

We will consider a system where the matter is an elementary Nambu-Goto string. Its action is given by

$$
I_{\text {matter }} \equiv I_{\text {string }}=-\mu \int d^{2} \sigma \sqrt{|\gamma|},
$$

where $\mu$ is the string tension and $\gamma$ is the determinant of the induced metric on the worldsheet:

$$
\gamma_{a b}(z)=g_{\mu \nu}(z) \partial_{a} z^{\mu} \partial_{b} z^{\nu} .
$$

In the presence of a string, the Lorentzian Einstein equations still admit ordinary black hole solutions corresponding to trivial string solutions spanning zero area. The continuation of these solutions to imaginary time is given by the metric

$$
d s^{2}=\left(1-\frac{2 M}{r}\right) d t^{2}+\left(1-\frac{2 M}{r}\right)^{-1} d r^{2}+r^{2} d \Omega^{2}
$$

for $r>2 M$. To determine the Euclidean manifold at $r=2 M$ we impose the Euclidean Einstein equations. As discussed in the Introduction, the free energy will then be computed on the saddle of the Euclidean action.

The action Eq. (17) with matter term Eq. (7) can be extended to Euclidean metrics. The subtracted Euclidean action reads

$$
\begin{aligned}
\widetilde{I}-\widetilde{I}_{0}= & -\frac{1}{16 \pi} \int_{\widetilde{M}} \sqrt{g} R+\frac{1}{8 \pi} \int_{\partial \widetilde{M}} \sqrt{h} K \\
& -\frac{1}{8 \pi} \int_{(\partial \widetilde{M})_{\infty}} \sqrt{h_{0}} K_{0}+\mu \int d^{2} \sigma \sqrt{\gamma} .
\end{aligned}
$$

Here the world sheet has the topology of a 2-sphere. Because the Euclidean black holes Eq. (9) have only one boundary, namely at infinity, we must take $\partial \widetilde{M}=(\partial \widetilde{M})_{\infty}$ in the $K$-term. The $K_{0}$-term subtraction has to be performed with the extrinsic curvature in flat Euclidean space. The independent variables in Eq. (10) are the components of the metric $g_{\mu \nu}$ and the 
string coordinates $z^{\mu}$. The variation of the action with respect to $g_{\mu \nu}$ gives the Euclidean Einstein equations:

$$
R_{\mu \nu}(x)-\frac{1}{2} g_{\mu \nu}(x) R(x)=8 \pi T_{\mu \nu}(x)
$$

where

$$
T^{\mu \nu}(x)=-\mu \int d^{2} \sigma \sqrt{\gamma} \gamma^{a b} \partial_{a} z^{\mu} \partial_{b} z^{\nu} \frac{1}{\sqrt{g(z)}} \delta^{4}(x-z) .
$$

Variations with respect to $z^{\mu}$ give rise to the stationary area condition for the string.

The Einstein equations Eq. (11) admit ordinary Euclidean black holes solutions corresponding to trivial zero string area. However the topology of the Euclidean manifold allows for a non-trivial string solution with minimal area which wraps around the continuation of the horizon $r=2 M$. All solutions are correctly described by the metric Eq. (9) but the non-trivial one has a curvature singularity at $r=2 M$. To analyze its structure, we write the metric in the vicinity of the horizon in terms of the proper radial length $\xi$ at fixed time $t$

$$
\left(1-\frac{2 M}{r}\right)^{-\frac{1}{2}} d r=d \xi \quad \xi=0 \quad \text { at } \quad r=2 M,
$$

which gives to order $\xi^{2}$

$$
d s^{2}=\xi^{2}\left(\frac{d t}{4 M}\right)^{2}+d \xi^{2}+r^{2} d \Omega^{2} .
$$

The Euclidean space is regular at $r=2 M$ when the periodicity of $t$ is

$$
\beta_{H}=8 \pi M
$$

and hence when it coincides with the inverse Hawking temperature $\beta_{H}^{-1}$ of the thermal flux emitted by the incipient black hole Eq. (1). This periodicity is modified by the presence of a string wrapped around the horizon. The trace of Einstein equations Eq. (11) gives

$$
\int_{\widetilde{M}} \sqrt{g} R=16 \pi \mu A
$$


where $A$, the area of the string, is equal to the area of the two sphere at $r=2 M$. The entire contribution to the integral comes from the string singularity. To evaluate $\int \sqrt{g} R$ when $R$ is zero everywhere except on the horizon, one can consider a tubular neighborhood $S^{2} \times D_{\epsilon}$ of $r=2 M$, of radial length $\epsilon$, and let $\epsilon$ tend to zero $[7,8]$. This gives

$$
\int_{S^{2} \times D_{\epsilon}} \sqrt{g} R=A \int_{D_{\epsilon}} \sqrt{(2)} g^{(2)} R .
$$

The Euler characteristic of the disc topology is $\chi=1$. It is given by

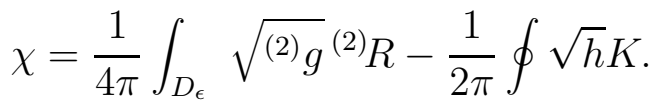

For a curvature singularity at the origin with

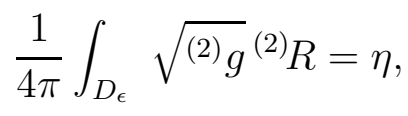

we find, from Eq. (18),

$$
-\oint \sqrt{h} K=2 \pi(1-\eta)
$$

This signals the existence of a conical singularity with deficit angle $2 \pi \eta$. Comparing Eq. (16) and Eq. (17), we then conclude that the string gives rise to a deficit angle

$$
\eta=4 \mu \text {. }
$$

This is the sole effect of the string instanton. From the explicit form of the Schwarzschild solution given in Eq. (14), we see that the periodicity of $t$ has become:

$$
\beta=\beta_{H}(1-4 \mu) .
$$

The string instanton has raised the global temperature from $\beta_{H}^{-1}$ to $\beta^{-1}$.

We now evaluate the free energy of the black hole. The contribution of the string term to the action Eq. (10) exactly cancels the contribution of the Einstein term as seen from Eq. (16). The boundary terms at asymptotically large $r=r_{\infty}$ are thus the only ones contributing to $\beta F$. Using Eqs. (5) and (9) we find

$$
\frac{1}{8 \pi} \int_{\partial \widetilde{M}=(\partial \widetilde{M})_{\infty}} \sqrt{h} K=-\beta\left(r_{\infty}\left(1-\frac{2 M}{r_{\infty}}\right)+\frac{M}{2}\right)
$$


The subtracted term is computed similarly in the flat metric

$$
d s^{2}=\left(1-\frac{2 M}{r_{\infty}}\right) d t^{2}+d r^{2}+r^{2} d \Omega^{2},
$$

where $t$ has the periodicity $\beta$ given by Eq. (22). The subtraction term is

$$
\frac{1}{8 \pi} \int_{(\partial \widetilde{M})_{\infty}} \sqrt{h_{0}} K_{0}=-\beta r_{\infty}\left(1-\frac{2 M}{r_{\infty}}\right)^{\frac{1}{2}} .
$$

The free energy is given by

$$
F(\beta, \mu)=\beta^{-1}\left(\widetilde{I}-\widetilde{I}_{0}\right)_{\text {saddle }}=\frac{M}{2} .
$$

Note that the free energy has the same value it had in the absence of the string instanton. However the thermodynamic potential $F$ is now a function of $\beta$ and the external parameter $\mu$. The entropy is given by:

$$
S=\left.\beta^{2} \frac{\partial F}{\partial \beta}\right|_{\mu}=\left.\frac{\beta^{2}}{2} \frac{\partial M}{\partial \beta}\right|_{\mu}=\frac{\beta^{2}}{16 \pi(1-4 \mu)} .
$$

Using Eq. (22) we find

$$
S=(1-4 \mu) \frac{A}{4} .
$$

This was the central result of reference [4]. The introduction of the external parameter $\mu$ has enabled us to define a black hole with fixed mass $M$ at a temperature other than the usual $\beta_{H}^{-1}$. When the temperature is not the Hawking temperature, the entropy changes from $A / 4$ to Eq. (28). It follows from Eq. (22) and Eq. (28) that the product of the entropy and the temperature is constant for a given mass, independent of the string tension

$$
\beta^{-1} S=\beta_{H}^{-1} S_{H}=\frac{M}{2},
$$

where $S_{H}=A / 4$.

To complete the thermodynamic analysis, we first verify using Eqs.(29) and (26) that the energy of the solution is unchanged by the presence of the string instanton:

$$
E=F+\beta^{-1} S=\frac{M}{2}+\beta_{H}^{-1} \frac{A}{4}=M .
$$


We immediately conclude from this equation that the identity

$$
\begin{aligned}
d M & =\beta_{H}^{-1} d S_{H} \\
& =\beta^{-1} d S+\beta^{-1} A d \mu,
\end{aligned}
$$

is nothing but an expression of the first principle of black hole thermodynamics in the presence of a string.

The area entropy of an eternal black hole can thus vary from the standard value $A / 4$ to 0 as a function of the string tension. All these black holes differ only by the instanton effect. One cannot distinguish them by their mass or even by their Lorentzian metrics. They differ through quantum effects, not in classical quantities such as $M / 2$, the product of $\beta^{-1}$ and $S$. Indeed the temperature is proportional to $\hbar$ while the entropy is inversely proportional to $\hbar$. In this sense the instanton provides a quantum hair [5, 6] which affects the expectation values of operators outside the horizon. Quantum effects will be further analyzed in Section 5 .

The above result rely crucially on the working hypothesis that the free energy of the Schwarzschild background is obtained from the saddle of the Euclidean functional integral at analytically continued solutions of the Einstein equation defining the Lorentzian background. We have also assumed that continuations corresponding to different instantons, and in particular to the trivial one with $\beta=\beta_{H}$, generate distinct black holes. The discussion of the validity of these assumptions will be the object of Section 1 . Before confronting the issue however, we shall establish the general nature of the entropy Eq. (28) emerging from this working hypothesis.

\section{The Black Hole in Presence of Classical Matter}

We now show that the entropy Eq. (28) is a genuine property of the horizon. Indeed we shall prove that this result is not affected by classical static matter outside the horizon. Classical surrounding matter does change the temperature but does not affect the entropy.

We surround the eternal black hole by a static and regular distribution of matter which is a spherically symmetric solution of Einstein equations for a 
coupled gravity-matter system. The new matter action in Eq. (4) now reads

$$
I_{\text {matter }}=I_{\text {string }}+I_{\text {matter }}^{\prime} .
$$

For $r>2 M$, the Euclidean metric solution of the Euclidean Einstein equations is given by

$$
d s^{2}=g_{t t} d t^{2}+g_{r r} d r^{2}+r^{2} d \Omega^{2}
$$

with

$$
\begin{aligned}
& g_{t t}(r)=\left(1-\frac{2 M(r)}{r}\right) \exp \left[-8 \pi \int_{r}^{\infty}\left(-T_{t}^{t}\left(r^{\prime}\right)+T_{r}^{r}\left(r^{\prime}\right)\right) r^{\prime} g_{r r}\left(r^{\prime}\right) d r^{\prime}\right] \\
& g_{r r}(r)=\left(1-\frac{2 M(r)}{r}\right)^{-1}
\end{aligned}
$$

where

$$
M(r)=M+\int_{2 M}^{r} 4 \pi r^{2}\left(-T_{t}^{t}\left(r^{\prime}\right)\right) d r^{\prime},
$$

and $M$ is still the black hole mass. The regularity condition characterizing the matter is $-T_{t}^{t}(2 M)+T_{r}^{r}(2 M)=0$. We parametrize again the metric in the vicinity of the horizon in terms of the proper length $\xi$ at fixed time $t$. Noticing that $g_{t t}=O\left(\xi^{2}\right)$ we find

$$
d s^{2} \simeq \xi^{2}\left(\frac{d t}{\lambda(0)}\right)^{2}+d \xi^{2}+r^{2} d \Omega^{2}
$$

where in general

$$
\lambda(\xi) \equiv \frac{d g_{t t}^{\frac{1}{2}}}{d \xi}=\frac{1}{2}\left(g_{t t} g_{r r}\right)^{-\frac{1}{2}} \frac{d g_{t t}}{d r} .
$$

This equation replaces Eq. (14) in the presence of matter. The deficit angle engendered by the string is still given by $2 \pi \eta=8 \pi \mu$ since the matter distribution, which is regular, affects Eq. (17) only to order $\epsilon^{2}$ and hence vanishes in the limit $\epsilon \rightarrow 0$. The inverse temperature of the black hole surrounded by regular static matter thus becomes

$$
\beta=\beta_{H m}(1-4 \mu),
$$

\footnotetext{
${ }^{5}$ The possible instabilities in the static matter distribution can always be compensated by external forces.
} 
where

$$
\beta_{H m}=\frac{2 \pi}{\lambda(0)}
$$

is the Hawking inverse temperature in the presence of matter (see for instance references 12, 13, 14).

We now compute the free energy from the Euclidean action. The new subtracted Euclidean action can be evaluated on the solution of the equations of motion

$$
\begin{aligned}
\widetilde{I}^{\prime}-\widetilde{I}_{0}^{\prime}= & -\frac{1}{16 \pi} \int_{\widetilde{M}_{\epsilon}} \sqrt{g} R+\mu \int d^{2} \sigma \sqrt{\gamma} \\
& -\frac{1}{16 \pi} \int_{\widetilde{M} \backslash \widetilde{M}_{\epsilon}} \sqrt{g} R+\widetilde{I}_{\text {matter }}^{\prime} \\
& +\frac{1}{8 \pi} \int_{(\partial \widetilde{M})_{\infty}}\left(\sqrt{h} K-\sqrt{h_{0}} K_{0}\right)
\end{aligned}
$$

where $\widetilde{M}$ denotes the Euclidean manifold of the solution and $\widetilde{M}_{\epsilon}=S^{2} \times D_{\epsilon}$ is a tubular neighborhood of the horizon of length $\epsilon$. The first two terms in Eq. (41) cancel each other as $\epsilon \rightarrow 0$ while $\widetilde{I}_{\text {matter }}^{\prime}$ is equal for this static solution to $\beta H_{\text {matter }}^{\prime}$ where $H_{\text {matter }}^{\prime}$ is the Hamiltonian constructed from $I_{\text {matter }}^{\prime}$. The surface terms

$$
\frac{1}{8 \pi} \int_{(\partial \widetilde{M})_{\infty}}\left(\sqrt{h} K-\sqrt{h_{0}} K_{0}\right)=\beta \frac{M_{t o t}}{2},
$$

are obtained by substituting in Eqs. (23) and (25) $M$ by $M_{t o t}=M\left(r_{\infty}\right)$. Thus Eq. (41) yields

$$
F=-\frac{1}{16 \pi} \int_{\left(\widetilde{M} \backslash \widetilde{M}_{\epsilon}\right)_{t}} \sqrt{g} R+H_{\text {matter }}^{\prime}+\frac{M_{t o t}}{2}
$$

where the $t$-subscripts indicate that the integrals are carried over the three dimensional static domain obtained by intersecting $\widetilde{M} \backslash \widetilde{M}_{\epsilon}$ by a constant $t$ plane. In order to obtain a simpler expression for $F$, we start from the Hamiltonian constraint, integrated over $\left(\widetilde{M} \backslash \widetilde{M}_{\epsilon}\right)_{t}$

$$
-\frac{1}{16 \pi} \int_{\left(\widetilde{M} \backslash \widetilde{M}_{\epsilon}\right)_{t}} \sqrt{g}^{(3)} R+H_{\text {matter }}^{\prime}=0
$$

where ${ }^{(3)} R$ is the three dimensional curvature of $\left(\widetilde{M} \backslash \widetilde{M}_{\epsilon}\right)_{t}$. Expressing ${ }^{(3)} R$ in terms of $R$,

$$
\frac{1}{16 \pi} \int_{\left(\widetilde{M} \backslash \widetilde{M}_{\epsilon}\right)_{t}}\left(\sqrt{g} R-\sqrt{g}{ }^{(3)} R\right)=-\frac{1}{8 \pi} \int_{\left(\widetilde{M} \backslash \widetilde{M}_{\epsilon}\right)_{t}} \partial_{i}\left(\sqrt{{ }^{(3)} g} g^{i j} \partial_{j} g_{t t}^{\frac{1}{2}}\right)
$$


where $g_{i j}$ is the metric tensor of the three surface, we recover the integral mass formula 11, 16, 17, 12, 13.

$$
-\frac{1}{16 \pi} \int_{\left(\widetilde{M} \backslash \widetilde{M}_{\epsilon}\right)_{t}} \sqrt{g} R+H_{\text {matter }}^{\prime}+\beta_{H m}^{-1} \frac{A}{4}-\frac{M_{t o t}}{2}=0 .
$$

The free energy is then obtained by comparing Eqs. (43) and (46):

$$
F=M_{t o t}-\beta_{H m}^{-1} \frac{A}{4} \text {. }
$$

To compute the entropy, we need to take the variation of $F$, hence the variation of $M_{t o t}$. Our starting point will be the integral mass formula Eq. (46). Notice that its form is identical in the Euclidean or Lorentzian formulation. In the latter case the integral is carried over a three dimensional static domain which is the same as in the Euclidean case. We thus skip the "tilde" notation and consider the formula in the limit $\epsilon \rightarrow 0$

$$
\lim _{\epsilon \rightarrow 0}\left(-\frac{1}{16 \pi} \int_{\left(M \backslash M_{\epsilon}\right)_{t}} \sqrt{|g|} R\right)+H_{\text {matter }}^{\prime}+\beta_{H m}^{-1} \frac{A}{4}-\frac{M_{t o t}}{2}=0 .
$$

We now vary Eq. (48) along the space of spherically symmetric static solutions [12, 13]. We multiply Eq. (48) by a finite time interval $\Delta t$ and replace $\Delta t H_{\text {matter }}^{\prime}$ by $-I_{\text {matter }}^{\prime}$. The variation of the two first terms in the fixed finite time interval vanishes on the space of solutions except for the boundary terms of the Einstein-Hilbert action and for the variation of external nongravitational parameters in the matter action. The former is:

$$
\begin{aligned}
\frac{1}{\Delta t} \delta\left(\frac{1}{16 \pi} \int_{M} \sqrt{|g|} R\right) & =\frac{1}{16 \pi \Delta t} \int_{M}\left(\partial_{\eta}\left(\sqrt{|g|} g^{\mu \nu} \delta \Gamma_{\mu \nu}^{\eta}\right)-\partial_{\nu}\left(\sqrt{|g|} g^{\mu \nu} \delta \Gamma_{\mu \eta}^{\eta}\right)\right) \\
& =\frac{1}{2}(2 M)^{2} \delta \lambda(0)-\left[\frac{1}{2} r^{2} \delta \lambda+r\left(-g_{t t}\right)^{\frac{1}{2}} \delta g_{r r}^{-\frac{1}{2}}\right]_{r_{\infty}} \\
& =\frac{A}{4} \delta \beta_{H m}^{-1}+\frac{\delta M_{t o t}}{2} .
\end{aligned}
$$

The evaluation of the right hand side of the above equation is straightforward using the proper length variable $\xi$. In Eq. (49), $\lambda(0)$ is given by Eq. (40) and $\lambda\left(r_{\infty}\right)=M_{t o t} / r_{\infty}^{2}$ is evaluated using Eq. (38). Inserting Eq. (49) in the variation of Eq. (48), gives the differential mass formula [16] in the form [12, 13]:

$$
\delta M_{t o t}=\beta_{H m}^{-1} \frac{\delta A}{4}+\delta_{e x t} H_{m a t t e r}^{\prime} .
$$


Here $\delta_{\text {ext }} H_{\text {matter }}^{\prime}$ results from the variation of all parameters in the matter Hamiltonian outside the horizon. Using now Eq. (50), and taking the variation with respect to $\beta$ with the other external parameters kept fixed, we find

$$
S=\left.\beta^{2} \frac{\delta F}{\delta \beta}\right|_{\mu, e x t}=-\left.\beta^{2} \frac{A}{4} \frac{\delta \beta_{H m}^{-1}}{\delta \beta}\right|_{\mu}=(1-4 \mu) \frac{A}{4} .
$$

We find that the entropy of the black hole, as in the absence of classical surrounding matter, is given by Eq. (28). The generality of this result shows that the area entropy in presence of a string instanton is independent of the classical matter content outside the black hole. This result is consistent with the fact that there is no tree level contribution to the entropy of the matter outside the black hole. Eq. (29) generalizes to

$$
\beta^{-1} S=\beta_{H m}^{-1} \frac{A}{4}
$$

By performing a Legendre transform, we find, as before, that the energy of the solution is unchanged by the presence of the instanton:

$$
E=F+\beta^{-1} S=M_{t o t}
$$

\section{The Significance of the Euclidean Saddle}

As stated in the Introduction, the canonical partition function summed over all black hole configurations diverges if the exponential of the area entropy is identified with the degeneracy of the corresponding black hole background. The elimination of the divergence by restricting the summation to the saddle contribution of the Euclidean action is not an a priori legitimate procedure. In this Section we shall first give a thermodynamic justification of the saddle prescription. We will then recall [18 how the instability arising in the canonical ensemble approach is avoided in the microcanonical ensemble. It will then be clear that black holes of the same mass but with different Euclidean sections have to be considered as thermodynamically distinct.

We first prove that the saddle prescription follows from thermodynamics if we assume that $M_{t o t}$ represents the thermodynamic energy and that the black hole generates entropy. Namely, we show that these assumptions require the introduction of an external parameter in the action (the string tension) 
when the temperature departs from the value $\beta_{H m}^{-1}$ and that the Euclidean periodicity must then be constrained by adjusting the Euclidean background to be a solution of the Euclidean equations of motion.

We start from the differential mass formula Eq. (50):

$$
\delta M_{t o t}=\beta_{H m}^{-1} \frac{\delta A}{4}+\delta_{e x t} H_{m a t t e r}^{\prime} .
$$

It is important to realize that the derivation of this identity involves only classical physics, is completely independent of any boundary terms which were added to the Einstein-Hilbert action, and does not appeal to Euclidean continuation.

If the system can be thermalized at an arbitrary temperature $\beta^{-1}$, the second term in the last equation can be rewritten as

$$
\delta_{\text {ext }} H_{\text {matter }}^{\prime}=\beta^{-1} \delta S_{\text {matter }}+\sum_{i} \partial_{\lambda_{i}} H_{\text {matter }}^{\prime} \delta \lambda_{i}
$$

The last term is the work done by the variation of the macroscopic parameters $\lambda_{i}$ in the action. The first term, where $S_{\text {matter }}$ is the matter entropy, would arise if some averaging over "microscopic" parameters had been performed, and is included here for the sake of generality. Notice that the volume is not a parameter but a dynamical quantity determined from the equation of motion. Inserting Eq. (55) back into Eq. (54) we get

$$
\delta M_{t o t}=\beta_{H m}^{-1} \frac{\delta A}{4}+\beta^{-1} \delta S_{\text {matter }}+\sum_{i} \partial_{\lambda_{i}} H_{\text {matter }}^{\prime} \delta \lambda_{i} .
$$

In order to interpret Eq. (56) as an expression of the first principle of thermodynamics we need the Bekenstein assumption [2] that the black hole contributes to the entropy. This assumption forces us to rewrite the first term as

$$
\beta_{H m}^{-1} \frac{\delta A}{4}=\beta^{-1} \delta S_{b h}+\sum_{i} X_{i} \delta \lambda_{i}
$$

where $X_{i}$ are generalized forces. Writing the temperature as $\beta=\beta_{H m}(1-\eta)$, one gets

$$
\beta_{H m}^{-1} \frac{\delta A}{4}=\beta^{-1} \delta S_{b h}+\beta^{-1} \frac{A}{4} \delta \eta,
$$


and

$$
\delta S_{b h}=\delta\left(\frac{A}{4}(1-\eta)\right) .
$$

Comparing Eq. (57) and Eq. (58), we learn that $\eta$ must be an external parameter contained in the list $\left\{\lambda_{i}\right\}$. The inverse temperature $\beta$ is equal to the period of the Euclidean continuation and $2 \pi \eta$ is therefore the deficit angle of the Euclidean manifold. Since the integral mass formula is valid independently of the value of the deficit angle, we must have $\partial_{\eta} H^{\prime}=0$. Had we not introduced a string term in the action we would not have been able to include $\eta$ in the set $\left\{\lambda_{i}\right\}$. The first principle would remain valid only at one value of the inverse temperature namely $\beta=\beta_{H m}$. In this particular case we recover of course all the known results. If, however, we introduce the string, $\eta$ must be a function of the string tension $\mu$.

We now show that thermodynamics imposes that this function be fixed by the Euclidean equations of motion. It is obvious that the static matter outside the horizon satisfies the Euclidean equations. What remains to be proven is that these are also verified at the Euclidean continuation of the horizon. We stress once more that Eq. (56) is classical and that each term must be independent of $\hbar$. Since the temperature has been seen to be proportional to $\hbar$, it follows, as expected, that $\delta S_{\text {matter }}$ vanishes along the space of classical solutions. Thus if the integration constant of the entropy is zero, the free energy of the system is

$$
\begin{aligned}
F & =M_{t o t}-\beta^{-1} S_{b h} \\
& =M_{t o t}-\beta_{H m}^{-1} \frac{A}{4} .
\end{aligned}
$$

The same value for the free energy Eq. (47) was deduced from the saddle point evaluation of the Euclidean action thereby fixing the value $\eta=4 \mu$ as in Eq. (21). If we had evaluated the free energy off-shell, we would not have been able to express the free energy in terms of only surface integrals and we would not have recovered the result Eq. (60). Note also that the value of the generalized force $\beta^{-1} A / 4$ appearing in Eq. (58) coincides with the one in Eq. (32) obtained from the functional integral and is not affected by the surrounding matter.

We now examine the consistency of the thermodynamics arising from the Euclidean saddle with the statistical interpretation in the microcanonical ensemble. Consider a system composed of gravity and matter fields. The 
classical solutions of the equations of motion define backgrounds for matter and gravitational quantum fluctuations. We shall neglect the latter and the backreaction of the matter field fluctuations on the geometry. We consider, within a finite volume $V$, spherically symmetric backgrounds with a black hole of mass $M$ dressed by a string instanton. The total entropy is a function of the total energy $E$ and of $V$. We write the total Hamiltonian as $H=$ $H_{g}+H_{\text {matter }}^{\prime}+H_{\text {matter }}^{f}$ where $H_{g}$ is the Hamiltonian of gravity and $H_{\text {matter }}^{f}$ is the Hamiltonian characterizing the fluctuating matter. Using Eq. (28) the total entropy $S_{\text {tot }}$ of the system can be expressed as

$$
\begin{aligned}
\exp S_{t o t}= & \operatorname{Tr} \delta(E-H) \\
= & \int_{0}^{+\infty} d M \int_{-\infty}^{+\infty} d t \exp \left[i t E+4 \pi M^{2}(1-4 \mu)-i t M_{t o t}\right] \\
& \operatorname{Tr}_{f} \exp \left[-i t H_{\text {matter }}^{f}\right] \\
= & \int_{0}^{+\infty} d M \int_{-\infty}^{+\infty} d t \exp \left[i t E+4 \pi M^{2}(1-4 \mu)-i t M_{t o t}-i t F_{f}(i t)\right]
\end{aligned}
$$

Here, the trace over matter states defining the free energy $F_{f}$ is taken in a background characterized by the black hole mass $M$ and the on-shell mass value $M_{\text {tot }}$ of the Hamiltonian $H_{g}+H_{\text {matter }}^{\prime}$. Evaluating the $t$-integral by the steepest descend method gives

$$
\begin{aligned}
\exp S_{t o t} & =\int_{0}^{+\infty} d M \exp \left[\beta_{f} E+4 \pi M^{2}(1-4 \mu)-\beta_{f} M_{t o t}-\beta_{f} F_{f}\left(\beta_{f}\right)\right] \\
& =\int_{0}^{+\infty} d M \exp \left[4 \pi M^{2}(1-4 \mu)+S_{f}\right]
\end{aligned}
$$

where $\beta_{f}$ is determined by the equation $E-M_{t o t}-\partial_{\beta_{f}}\left(\beta_{f} F_{f}\right)=0 . S_{f}$ is the entropy of the fluctuating matter in the thermodynamic limit of large $E$ and $E-M_{t o t}$ of order $E$. The saddle is a good one because the specific heat of the fluctuating matter is positive. $S_{t o t}$ is finally evaluated by taking in Eq. (62) the saddle in the $M$-integral. The saddle condition is:

$$
\frac{\partial S_{f}}{\partial M_{t o t}} \frac{\partial M_{t o t}}{\partial M}+8 \pi M(1-4 \mu)=0
$$

Equivalently,

$$
\frac{\partial S_{f}}{\partial M_{t o t}} 8 \pi M \beta_{H m}^{-1}+8 \pi M(1-4 \mu)=0
$$


where we used in Eq. (64) the differential mass formula Eq. (54). The Eq. (64) gives the equal temperature condition

$$
-\frac{\partial S_{f}}{\partial M_{t o t}}=\beta_{f}=\beta_{H m}(1-4 \mu)
$$

One easily verifies, when $H_{\text {matter }}^{f}$ describes a relativistic gas in absence of classical surrounding matter, that the saddle over $M$ is a maximum when the Hawking condition [18], $M>(4 / 5) E$, is satisfied, independently of the value of $\mu$. This saddle approximation is thus valid in the thermodynamic limit and the pathology of the canonical ensemble is avoided. We surmise that this remains true in general for regular distribution of matter.

Up to now, we have only considered string instantons which wrap once around the Euclidean 2-sphere $r=2 M$. The saddle point argument in the microcanonical ensemble gives back the temperature obtained from the saddle prescription in the canonical partition function. This confirms that the configuration with a string instanton has a well defined temperature different from the Schwarzschild black hole in the absence of string. One should consequently treat it as a distinct thermodynamical configuration with a lower entropy and higher temperature. One may also generate multiwrappings. These come about by mapping the world sheet, which was taken to have the topology of the sphere, onto an $n$-fold covering of the 2 -sphere $r=2 M$. Again, their temperatures, as given by the saddle point in the microcanonical ensemble, are distinct and define distinct thermodynamical configurations. It is easily verified that the entropy associated to an $n$-fold covering is

$$
S_{n}=(A / 4)(1-4 n \mu) .
$$

At this stage however, one should question the physical relevance of the latter result. Although these solutions are legitimate for free strings introduced in our action Eq. (10), such $n$-fold coverings is expected to render the result Eq. (66) sensitive to interactions.

\section{Quantum Matter on the Horizon}

The string instanton at $r=2 M$ in Euclidean space does not alter the classical Lorentzian black hole background which remains regular on the horizon. 
However dramatic effects occur at the quantum level. To illustrate these we consider the toy model consisting of the $s$-wave component of a free scalar field propagating on the Schwarzschild geometry and we neglect the residual relativistic potential barrier. This amounts to consider a 2-dimensional scalar field propagating on the radial subspace of the 4-geometry. We shall see that the string instanton induces, in an inertial frame, a singularity in the vacuum expectation value of the scalar field energy-momentum tensor on the horizon.

We parametrize the 2d-background using the light-like tortoise coordinates defined by

$$
u=t-r^{\star}, \quad v=t+r^{\star},
$$

where

$$
d r=\left(1-\frac{2 M}{r}\right) d r^{\star} \quad, \quad r^{\star}=-\infty \quad \text { at } \quad r=2 M
$$

and

$$
\begin{aligned}
d s^{2} & =-\exp (2 \rho) d u d v, \\
\exp (2 \rho) & =\left(1-\frac{2 M}{r}\right) .
\end{aligned}
$$

We compute now the expectation value of the energy-momentum tensor of the scalar field using the trace anomaly [19, 20]. Note that in the 2dimensional approximation the energy-momentum tensor of the quantum field $T_{\mu \nu}^{(2)}$ is related to the 4-dimensional one by $T_{\mu \nu}^{(2)}=4 \pi r^{2} T_{\mu \nu}$. Since in what follows, all the quantities are two-dimensional we shall drop the superscript (2).

The trace anomaly equation $\langle T\rangle=-R / 24 \pi$ yields in the metric Eq. (69)

$$
\left\langle T_{u v}\right\rangle=-\frac{1}{12 \pi} \partial_{u} \partial_{v} \rho
$$

From Eq. (71) and from the conservation law

$$
T_{\nu ; \mu}^{\mu}=0
$$

one gets

$$
\begin{aligned}
& \left\langle T_{u u}\right\rangle=\frac{1}{12 \pi}\left[-\frac{M}{2 r^{3}}\left(1-\frac{2 M}{r}\right)-\frac{M^{2}}{4 r^{4}}\right]+t_{u}(u) \\
& \left\langle T_{v v}\right\rangle=\frac{1}{12 \pi}\left[-\frac{M}{2 r^{3}}\left(1-\frac{2 M}{r}\right)-\frac{M^{2}}{4 r^{4}}\right]+t_{v}(v)
\end{aligned}
$$


where $t_{u}(u)$ and $t_{v}(v)$ are respectively arbitrary functions of $u$ and $v$.

The usual Hartle-Hawking vacuum is characterized by the regularity of $\left\langle T_{\mu \nu}\right\rangle$ on the past and future horizons in a local inertial frame. The latter can be parametrized by Kruskal coordinates $U$ and $V$ defined by

$$
\begin{aligned}
U & =-4 M \lambda e^{-\frac{u}{4 M}} \\
V & =4 M \lambda^{-1} e^{\frac{v}{4 M}},
\end{aligned}
$$

where $\lambda$ is an arbitrary boost parameter and $U=0$ and $V=0$ correspond respectively to the future horizon and the past horizon. Regularity in the $(U, V)$ frames implies that $\left\langle T_{u u}\right\rangle$ and $\left\langle T_{v v}\right\rangle$ vanish on these horizons like $O\left(\left(1-\frac{2 M}{r}\right)^{2}\right)$. This condition determines the arbitrary functions $t_{u}(u)$ and $t_{v}(v)$ to be constants and equal to

$$
t_{u}=t_{v}=\frac{\pi}{12} \frac{1}{(8 \pi M)^{2}}
$$

The $t_{u}$ term represents from Eq. (73) the outgoing flux as $r \rightarrow \infty$ and the $t_{v}$ term represents from Eq. (174) the ingoing flux as $r \rightarrow \infty$. Both of them together correspond to thermal equilibrium with the background at the Hawking temperature $\beta_{H}=8 \pi M$.

When the string instanton is present, the temperature gets modified according to Eq. (22). We may then characterize the new vacuum by taking

$$
t_{u}=t_{v}=\frac{\pi}{12} \frac{1}{(8 \pi M(1-4 \mu))^{2}} .
$$

Using Eqs. (73), (74), (78) and the coordinate transformation Eqs. (75), (76), we see that, in the local inertial frames, the components of the expectation values of the energy-momentum have singular parts on the horizons. They are given by

$$
\begin{aligned}
\left\langle T_{U U}^{\text {sing }}\right\rangle & =\frac{\mu}{6 \pi U^{2}} \frac{1-2 \mu}{(1-4 \mu)^{2}} \\
\left\langle T_{V V}^{\text {sing }}\right\rangle & =\frac{\mu}{6 \pi V^{2}} \frac{1-2 \mu}{(1-4 \mu)^{2}} .
\end{aligned}
$$

Eqs. (79) and (80) translate the fact that singularities occur on both horizons when thermal equilibrium in the Schwarzschild background is realized 
at a temperature different from the Hawking one. This feature should persist in more realistic four dimensional computations. Thus the string instanton amounts to a materialization of the horizon at the quantum level. Such a materialization constitute a non local effect whose origin can be traced to the non locality of the string.

The material horizon appears here as a property of quantum fields in thermal equilibrium in a black hole background. However a material future horizon could also emerge from the thermal radiation emitted from an incipient black hole. The point is that in four dimensions, the centrifugal barrier maintains thermal equilibrium in the vicinity of the horizon 21] and the Hawking radiation may be viewed, even in the conventional derivation, as a tiny disturbance from equilibrium due to the escape of the low angular momentum quanta out of the centrifugal barrier. This departure from thermal equilibrium becomes, close to the horizon, vanishingly small. Indeed, there, the local temperature tends to infinity and the fraction of angular momentum modes able to overcome the barrier becomes of zero measure [22]. Of course in the original derivation from local field theory, the global temperature describing this local equilibrium has the Hawking value Eq. (1) and no singularity will appears on the future horizon. But the huge energy fluctuations in vacuum close to the horizon renders local field theory vulnerable to Planckian quantum gravity effects. Taming these fluctuations by back reaction may require new elements such as those contained in the string theory approach and this might lead to materialization of the horizon. Such a phenomenon could have important consequences for solving the unitarity problem posed by black hole evaporation. In fact if unitarity is to be preserved in our universe, as suggested by 't Hooft [23], some kind of materialization appears unavoidable [23, 21, 24, 25]. The fact that the materialization mechanism encountered here is accompanied by a decrease of the area entropy of the black hole suggests indeed that it is operative in retrieving the information from the evaporating black hole.

\section{Acknowledgements}

We would like to thank R. Argurio, R. Brout and Ph. Spindel for valuable discussions. This work was supported in part by the Centre National de la Recherche Scientifique and the EC Science grant ERB 4050 PL920982. 


\section{References}

[1] S.W. Hawking, Commun. Math. Phys. 43 (1975) 199.

[2] J.D. Bekenstein, Phys. Rev. D7 (1973) 2333.

[3] G.W. Gibbons and S.W. Hawking, Phys. Rev. D15 (1977) 2752.

[4] F. Englert, L. Houart and P. Windey, The black hole entropy can be smaller than A/4, preprint PAR-LPTHE 95-11 and ULB-TH 05/95 (1995) hep-th/9503202.

[5] S. Coleman, J. Preskill and F. Wilczek,Nucl. Phys. B378 (1992) 175.

[6] F. Dowker, R. Gregory and J. Traschen, Phys. Rev. D45 (1992) 2762.

[7] M. Bañados, C. Teitelboim and J. Zanelli, Phys. Rev. Lett. 72 (1994) 957.

[8] C. Teitelboim, Phys. Rev. D51 (1995) 4315.

[9] S. Carlip and C. Teitelboim, Class. Quantum Grav. 12 (1995) 1699.

[10] L. Susskind and J. Uglum, Phys. Rev. D50 (1994) 2700.

[11] S.W. Hawking and G. Horowitz, The gravitational Hamiltonian, action, entropy and surface terms, preprint DAMTP/R 94-52 and UCSBTH94-37 (1995) gr-qc/9501014.

[12] A. Casher and F. Englert, Class. Quantum Grav. 9 (1992) 2231.

[13] A. Casher and F. Englert, Class. Quantum Grav. 10 (1993) 2479.

[14] M. Visser, Phys. Rev. D46 (1992) 2445.

[15] L. Smarr, Phys. Rev. Lett. 30 (1973) 71; 30 (1973) 521(E).

[16] J.M. Bardeen, B. Carter and S.W. Hawking, Commun. Math. Phys. 31 (1973) 161.

[17] R. Wald, General Relativity (University of Chicago Press, 1984) Appendix E. 
[18] S. W. Hawking, Phys. Rev. D13 (1976) 191.

[19] N.D. Birell and P.C.W. Davies, Quantum Field Theory in Curved Space (Cambridge University Press, 1982).

[20] P.C.W. Davies, S.A. Fulling and W.G. Unruh, Phys. Rev. D13 (1976) 2720.

[21] L. Susskind, L. Thorlacius and J. Uglum, Phys. Rev. D48 (1993) 3743.

[22] F. Englert, S. Massar and R. Parentani, Class. Quantum Grav. 11 (1994) 2919.

[23] G. 't Hooft, Nucl. Phys. B256 (1985) 727.

[24] G. 't Hooft, Horizon operator approach to black hole quantization, presented at "The Black Hole 25 Years After", Santiago, Chile (17-21 January 1994), preprint THU-94/02 gr-qc/9402037.

[25] F. Englert, Operator weak value and black hole complementarity, presented at "The Oskar Klein Centenary Symposium", Stockholm, Sweden (19-21 September 1994), preprint ULB-TH 03/95 gr-qc/9502039. 